\title{
Probing Wolf-Rayet wind structures: helping hands from WR+O binaries
}

\author{
Sergey V. Marchenko \\ Département de Physique, Université de Montréal, \\ Montréal, Québec, Canada
}

\begin{abstract}
For binaries with well-known orbital parameters we describe methods to restrict some characteristics of the WR star in the binary, such as $T_{\mathrm{e}}$ and $R_{\mathrm{WR}}(\tau \simeq 1)$. The presence of a hot, luminous companion helps to address the question of spherical symmetry of the WR wind and reveal the ionization stratification in the wind. Also, we discuss the restrictions which can be imposed on the wind velocity law $(\mathrm{s})$.
\end{abstract}

\section{Modeling: WR+O binaries or single Wolf-Rayet stars?}

Considering $\mathrm{WR}+\mathrm{O}$ binaries as a potential source of information for a fine tuning of the 'standard model' of WR winds, the one principal advantage of binaries over single stars is that binaries with well-known orbital parameters provide a measure of distance in absolute terms. This is of crucial importance for tying down the wind velocity law, the dimensions of the line formation regions, etc. The main handicap of binaries is that there are too few well-studied WR+O systems, which contrasts with the numerous apparently single WR stars frequently used for a statistically meaningful sampling. However, as I will show below, some useful and far-reaching conclusions can be drawn on a sufficiently detailed data set of even a sole binary star.

\section{Large-scale structure of the Wolf-Rayet wind}

Two stars are used most frequently in attempts to model the WR wind structure: the apparently single WR star EZ CMa (WR 6, WN5, Hillier 1987a,b; Schmutz 1997), and the close massive binary V444 Cyg (WR 139, WN5+O6VIII, Cherepashchuk et al. 1984; Pauldrach et al. 1985; Hamann \& Schwarz 1992; Auer \& Koenigsberger 1994; Antokhin et al. 1997). Spherical symmetry of the WR wind, the starting assumption in any modeling, seems to fail in the case of EZ CMa, where the wind is flattened (Schulte-Ladbeck 1994; Harries et al. 1998) and subjected to strong periodic variability (probably rotationallyinduced: Morel et al. 1997, 1998). On the other hand, the wind of the WR component in V444 Cyg shows no detectable deviations from spherical symmetry at least for lines of high ionization formed close to the star. Moreover, the hot and luminous $\mathrm{O}$ companion affects only He I and some He II lines formed in the WR wind, mainly via the wind-wind collision phenomena (Marchenko et al. 1994, 1997; Stevens \& Howarth 1999). The base of the wind, as seen through spectral lines with high ionization potential, remains practically unperturbed (Fig. 1), thus providing a solid ground for the spherically symmetric approach. 


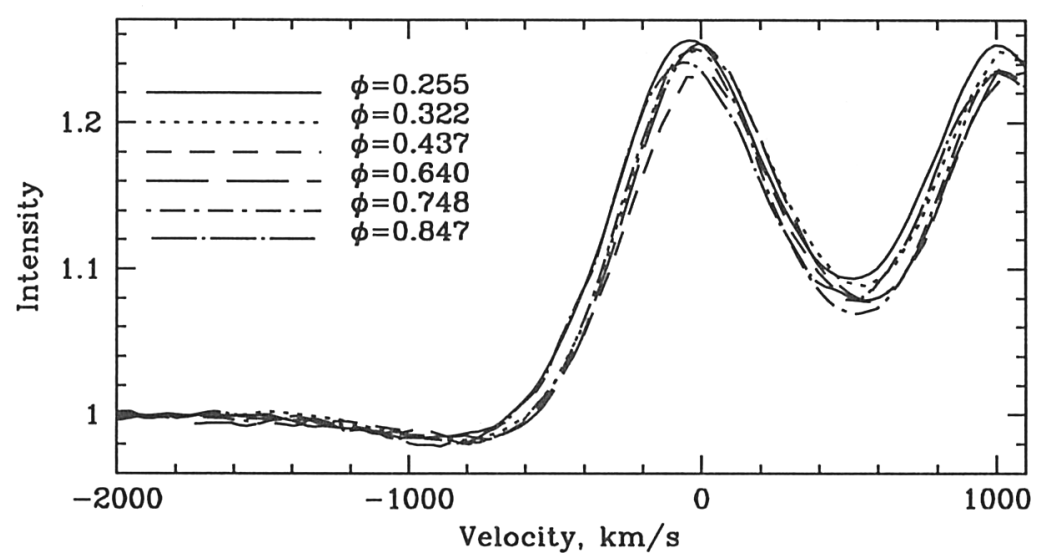

Figure 1. V444 Cygni. Line profiles of N v 4604,4620 for different orbital phases (WR star in front at $\phi=0$, cf. Marchenko et al. 1997).

\section{Constraints on $R_{W R}$ and $T_{e}$ in the Wolf-Rayet wind}

The orbital separation of the components in a binary system provides firm upper limits on their radii, with the best current examples the shortest-period binaries WR 46, Cyg X-3 and CQ Cep (Cherepashchuk \& Moffat 1994; Moffat \& Marchenko 1996; however, note that WR 46 could belong to to the V Sagittae group of variables: Steiner \& Diaz 1998). Much more accurate constraints can be obtained via interpretation and modeling of: (i) light curves (here I cite only the papers related to V444 Cyg: Kron \& Gordon 1950; Cherepashchuk et al. 1984; Underhill \& Fahey 1987; Antokhin et al. 1997); (ii) polarimetric data (Robert et al. 1990; St-Louis et al. 1993a; Rodrigues \& Magalhães 1995); and (iii) spectroscopy (Moffat \& Marchenko 1996). The last approach is practically assumption-free and deals with variations of the absorption troughs of P-Cygni profiles caused by eclipsing of the WR star by the $\mathrm{O}$ companion. Promising candidates to refine and apply this method are: WR 151, WR 155, HD 5980. Finally, for V444 Cyg we can claim a sound consensus on the estimated radii: $R_{\mathrm{WR}}=3.0 \pm 0.5 \mathrm{R}_{\odot}, R_{\mathrm{O}}=8.8 \pm 0.5 \mathrm{R}_{\odot}$, with only two notable deviations (Underhill \& Fahey 1987; Hamann \& Schwarz 1992). Here, $R_{\text {WR }}$ defines the radius of the WR star roughly corresponding to the $\tau \simeq 1$ 'event horizon' in the optically thick WR wind continuum.

An elegant method for estimation of the electron temperature in the WR wind was devised by Münch (1950), exploring the idea that the spectral features of the O-type component should be perceptibly broadened by the scattering on free electrons in the WR wind at phases when the $\mathrm{O}$ star passes behind the WR component. Applying this method to the high quality time-resolved spectra of V444 Cyg (Marchenko et al. 1997), we obtained $T_{\mathrm{e}}=11.5 \pm 2.5 \mathrm{kK}$ at $r \gtrsim 15 \mathrm{R}_{\odot} \simeq 5 \mathrm{R}_{\mathrm{WR}}$ distance from the WR star. There are two main deficiencies in the methodology: (a) the big size of the 'probe', the $O$ star; and $(b)$ the assumption that the bulk motion of the WR wind is negligible compared to the thermal motion of free electrons, which is only partially true. Incorporation 


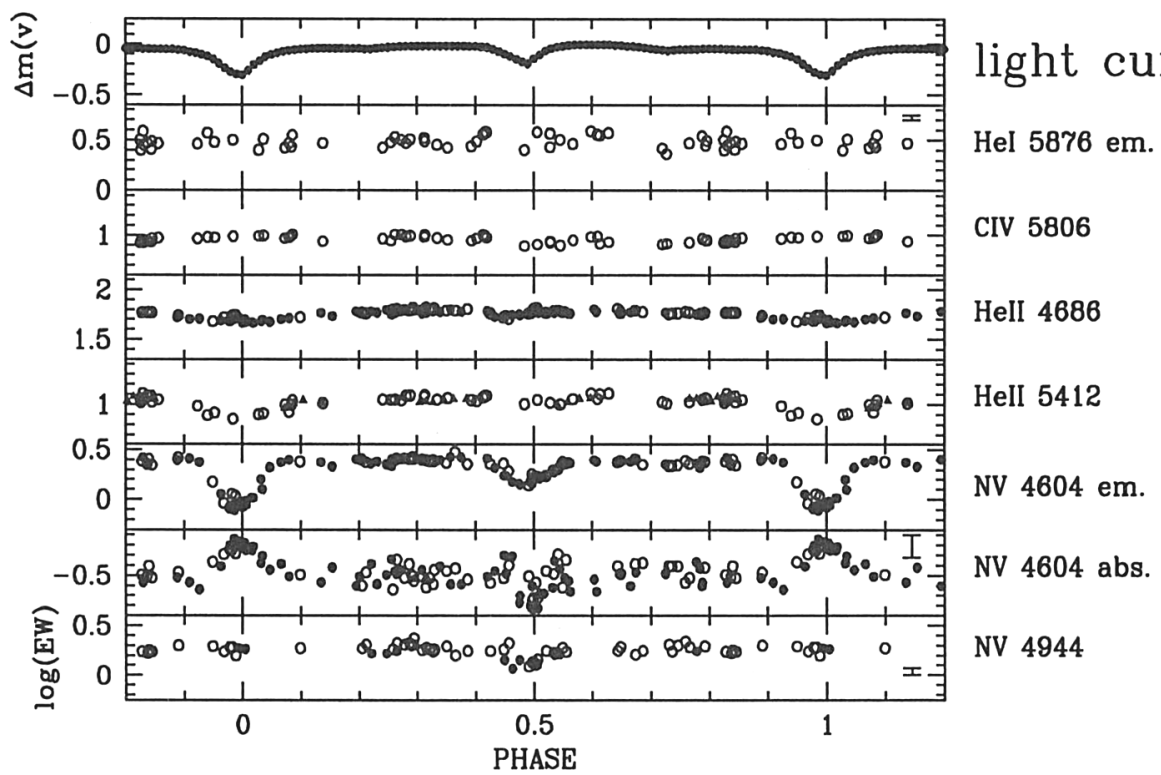

Figure 2. Phase dependence of equivalent widths in V444 Cygni. Filled dots: SPM Observatory (Mexico); open dots: OMM (Québec); triangles: CFHT (Hawaii). $2 \sigma$ error bars are shown for He I 5876, N v 4944 and absorp-

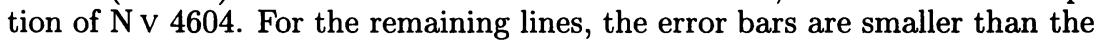
symbol sizes.

of detailed calculations in the frame of the 'standard model' may substantially improve sensitivity and accuracy of the diagnostics.

\section{Ionization stratification and atmospheric eclipses}

One way to approach the problem of ionization stratification in the WR wind is to use the traditional approach of establishing a dependence of WR line widths on ionization potential (Kuhi 1973; Schulte-Ladbeck et al. 1995; Rochowicz 1997). However, besides serving as a firm proof of ionization stratification, the approach provides no means for estimating the absolute dimensions of the line formation zones. This can be remedied by investigating the line-profile variations caused by eclipsing effects in $\mathrm{WR}+\mathrm{O}$ binaries. In Fig. 2 we plot the phase dependence of the equivalent widths in V444 Cyg, after correcting the data for the variable continuum level ( $c f$. Marchenko et al. 1997). The lines are arranged in accordance to their excitation potential (higher level). Taking into account both the shape and duration of the primary $(\phi=0$, WR star in front) and the secondary ( $\phi=0.5, \mathrm{O}$ star in front) minimum, we can readily conclude that the $\mathrm{CIV}$ and He I lines are formed in a big volume, $r \gtrless a$ (orbital separation), and $\mathrm{N} \mathrm{V}$ originates in the vicinity of the WR core. Also note that: (a) the minimum in the N v 4604 absorption at $\phi \simeq 0.5$ provides a possibility to estimate the radii of the components (Moffat \& Marchenko 1996); (b) the small reversal at $\phi \simeq 0$ in $E W(\mathrm{He}$ II 4686) signifies that the line is formed in a 'shell' 


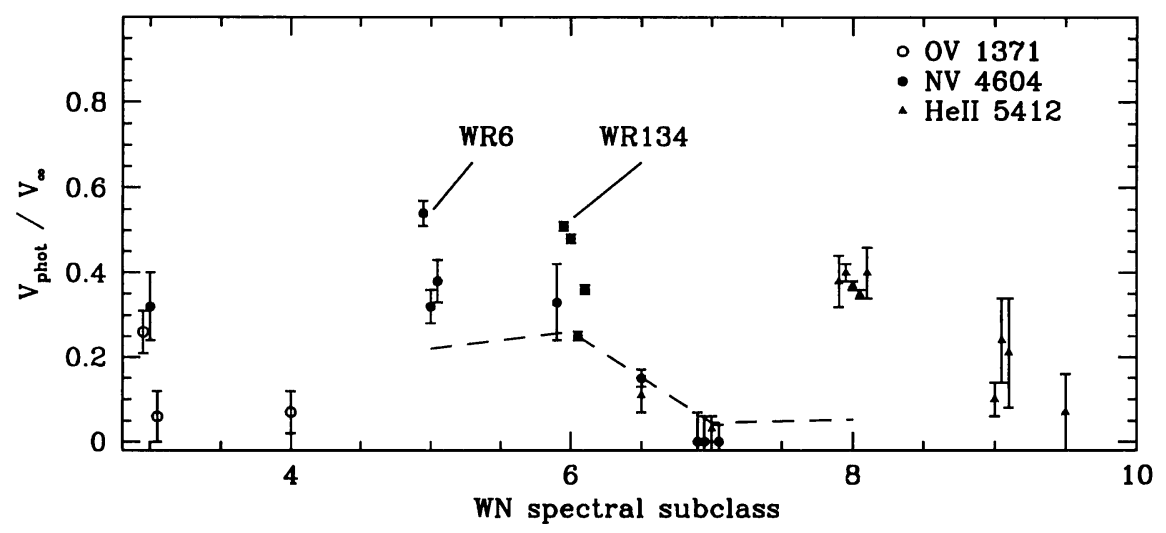

Figure 3. Spectral dependence of the $v_{\text {phot }} / v_{\infty}$ ratio for WN stars. The broken line indicates the result of modeling. The plotted $2 \sigma$ error bars account for uncertainties in $v_{\text {phot }}$ and $v_{\infty}$.

(cf. Eaton et al. 1985); and (c) the absence of primary eclipse in N v 4944 and C IV 5806 is a potential indicator of a high-intensity UV radiation field in the proximity of the WR core (cf. Hillier 1988).

Passage of a WR star in front of a luminous O companion may cause deep atmospheric (or selective wind) eclipses, if the orbital inclination of the system is high enough (good example: V444 Cyg around $\phi \simeq 0$; see also St-Louis et al. 1993b; Auer \& Koenigsberger 1994 and references therein). This is a relatively untapped source of information about the WR wind structure. So far, research was predominantly concentrated on UV data (chiefly from IUE), usually plagued by an inadequately low $\mathrm{S} / \mathrm{N}$. Surprisingly little work has been carried out in the optical. It is worth mentioning one particularly interesting result (Koenigsberger \& Auer 1985): the atmospheric eclipses observed in the N IV 1719 line in four different binaries bear the same kind of phase dependence, suggesting striking similarity from star to star of the WN wind structure. Summarizing all available data, one can draw the following conclusions about dimensions of the line formation zones:

N V 4603 is formed completely inside $r=15-20 \mathrm{R}_{\odot}$;

N IV 7112 inside $r \simeq 10 \mathrm{R}_{\odot}$;

Fe VI mainly inside $r=15-20 \mathrm{R}_{\odot}$, with rapid fall out to $r \simeq a$;

Fe $\mathrm{V}$ inside $r \simeq a$;

He II $+\mathrm{H} \alpha 6560$ inside $r \simeq a$;

He II 1640, N IV 1718 outside $r=10-15 \mathrm{R}_{\odot}$;

Si IV 1400 , He II 4686 at $r \simeq a$ and probably beyond, for a typical $a \simeq 40-60 \mathrm{R}_{\odot}$.

\section{Wind velocity law(s)}

Can we somehow estimate the efficiency of acceleration in the optically thin part of the WR wind? Under the assumption of monotonically, radially increasing 


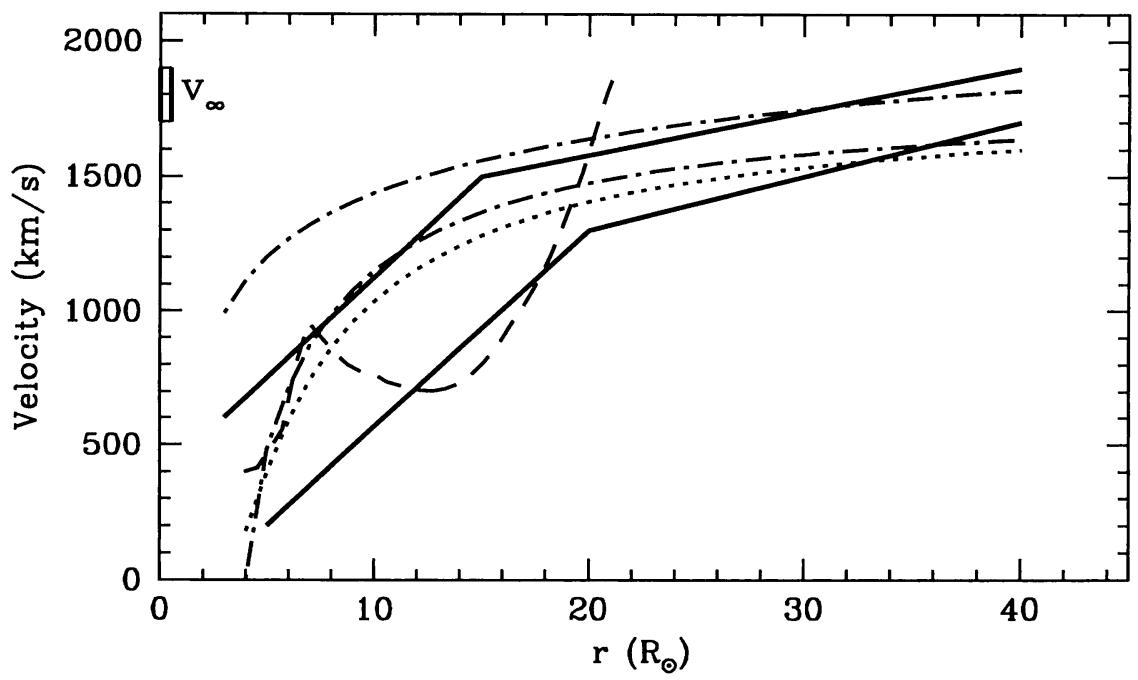

Figure 4. V444 Cygni. Thick lines: observationally imposed limits on the WR wind velocity law (spectroscopy); dotted line: $\beta=1.2$ law (Marchenko \& Moffat 1998a, profile fitting); dashed line: Antokhin et al. (1997, light curve solution); dot-dash lines: Springmann (1994, theory).

wind velocity, the efficiency will be related to the velocity corresponding to the transition between optically thick and optically thin parts of the wind. Naturally, this transition velocity should have strong wavelength dependence (Fig. 3 from Schmutz 1997). Nevertheless, we may place an upper limit on it by choosing the 'best' optical and UV lines ( $c f$. Marchenko \& Moffat 1998a). For the WN stars we find (Fig. 3): $v_{\text {phot }} / v_{\infty}=0.26 \pm 0.17$, where the 'starting' (i.e., at the inner boundary of the optically thin part of the wind) velocity of the wind is termed as $v_{\text {phot }}$, and the wind terminal velocity as $v_{\infty}$. This signifies that at least $3 / 4$ of the acceleration is exerted on a WN wind in its optically thin part. The most notable exceptions from the general $v_{\text {phot }} / v_{\infty}$ dependence are the stars with structured winds (EZ CMa: Morel et al. 1997, 1998; WR 134: Morel et al. 1999), and the 'violently'-variable WN8 stars (Marchenko et al. 1998).

The best method to unveil the wind velocity law is to perform an analysis of atmospheric eclipses, combining the resonance lines in the UV (large distances from the core) and the subordinate optical transitions (compact and intermediate-size line formation zones). For now, only rough constraints can be placed on the velocity law in the case of the best studied binary V444 Cyg (Fig. 4). In this figure, I incorporate: the information about the WR core size at $\tau \simeq 1$ in the optical (Moffat \& Marchenko 1996); the radial dependence of the optical depth in the Nv 4604 line (Marchenko et al. 1997); the conclusion that the wind terminal velocity is reached inside the $\mathrm{O}$ star orbit (Cherepashchuk et al. 1984; Auer \& Koenigsberger 1994). The traditional $\beta$-velocity law, $v(r)=v_{\text {phot }}+\left(v_{\infty}-v_{\text {phot }}\right)\left(1-R_{\mathrm{WR}} / r\right)^{\beta}$, with $\beta=1.2$ (Fig. 4$)$, provides good fits to some optical lines in V444 Cyg (mainly formed at $r \lesssim a$ : Marchenko \& Moffat 1998a). However, there is a systematic deviation from the $\beta$ law at $r \not 3 / 4 a$, 


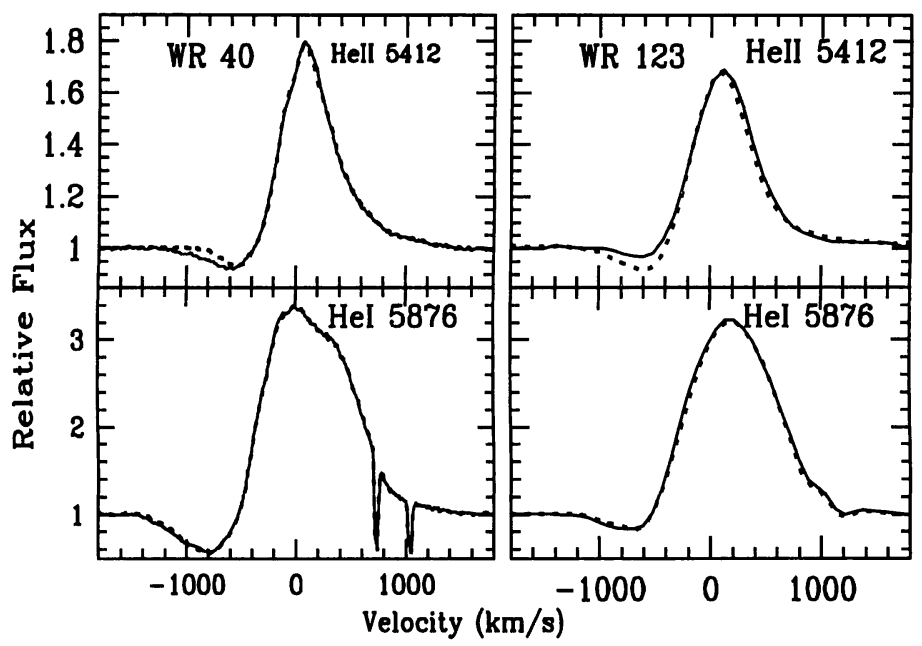

Figure 5. Short-term He II variations in WR 40 and WR 123. The profiles indicated by a dotted line were obtained $2-4 \mathrm{~h}$ after the full-line profiles. Note the lack of variations in $\mathrm{He}$ I at the given velocity in the variable $\mathrm{He}$ II profile.

which increases with distance, indicating that significant and extended wind acceleration may occur at large distances from the WR core, $r>10 R_{\mathrm{WR}}$.

The observational proof of any non-monotonic character of the wind velocity law is a task of utmost difficulty if we were to deal with a single star (note, however, some progress in a theoretial approach to the problem: Ignace et al. 1998). This might prove to be routinely simple for $\mathrm{WR}+\mathrm{O}$ binaries, with well documented atmospheric eclipses. Turning to single stars, we mention only one quite speculative case which might discern the non-monotonic character of the WR wind acceleration. Observing short-term (hours) line profile variations in the WN8 stars (Marchenko et al. 1998), we detected a very strange lack of synchronization in the variations of the He II vs. He I lines (Fig. 5), with the only plausible explanation as due to a non-monotonic velocity law. Note that we observed the variations in the absorption troughs, which practically eliminates the ambiguity arising from the line-of-sight projection of the observed wind velocities.

\section{6. 'Probing' instabilities}

Exploring the subject of intrinsic wind variability, it appears to be a powerful alternative method for probing WR wind structure by following the propagation of WR core-inflicted perturbations in the WR wind. The potential of this approach may be realised in full once one knows: $(a)$ the origin and the nature of the perturbation; $(b)$ the exact places where the propagating perturbations happened to be registered. The first condition may be readily fulfilled for single stars, with several good examples: EZ CMa, where the initial, core-generated periodic flux modulation triggers spectacular line profile variations (St-Louis et al. 1995; Morel et al. 1997, 1998); apparently single WN8 stars, where, by analogy 


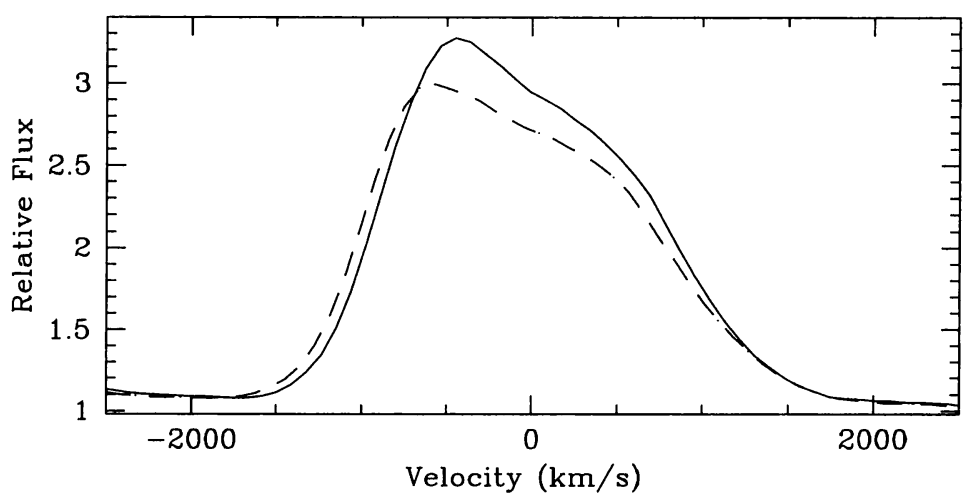

Figure 6. Rapid stochastic variability of the He II 4686 line in V444 Cygni. The profiles representing two extrema of the line variability are corrected for orbital shift and variable continuum level. The full line represents the mean of three spectra at $\phi=0.534,0.541,0.552$, all in different cycles; the dashed line represents the mean of four spectra at $\phi=0.520,0.549,0.559,0.562$, all in different cycles.

to EZ CMa, the variable stellar core drives the profile variability (Marchenko et al. 1998; Marchenko \& Moffat 1998b). The only substantial difference of the WN8 stars from EZ CMa is that the variability at times appears as purely stochastic, and at times may be represented by a multi-component frequency spectrum. Apparently, the second condition can be realised only in binaries with well-known orbital parameters.

One intriguing example is the variability observed in the He II 4686 emission in V444 Cyg (Fig. 6) around orbital phases $\phi=0.5-0.6$, when the line of sight is close to the axis of symmetry of the bow shock (Marchenko et al. 1997) formed in the vicinity of the $\mathrm{O}$ component, at $\Delta r \lesssim 1 R_{\mathrm{O}}$ from the surface. As we know the time and the position, we may proceed with interpretation, linking these variations to changes in the mass loss rate of the WR star. Such $\dot{M}$ fluctuations may be a prerequisit for growth of 'mechanical' instabilities (Stone et al. 1995), capable of operating at large $\left(r \gg 10 R_{\mathrm{WR}}\right)$ distances from the stellar core (see also Grosdidier et al. 1998).

Acknowledgments. I thank NSERC (Canada), IAU and IA UNAM (Mexico) for financial assistance.

\section{References}

Antokhin, I.I., Cherepashchuk, A.M., Yagola, A.G. 1997, ApSS 254, 111

Auer, L.H., Koenigsberger, G. 1994, ApJ 436, 859

Cherepashchuk, A.M., Moffat, A.F.J. 1994, ApJ 424, L53

Cherepashchuk, A.M., Eaton, J.A., Khaliullin, Kh.F. 1984, ApJ 281, 774

Eaton, J.A., Cherepashchuk, A.M., Khaliullin, Kh.F. 1985, ApJ 297, 266

Grosdidier, Y., Moffat, A.F.J., Joncas, G., Acker, A. 1998, ApJ 506, L127

Hamann, W.-R., Schwarz, E. 1992, A\&A, 261, 523 
Harries, T.J., Hillier D.J., Howarth, I.D. 1998, MNRAS 296, 1072

Hillier, D.J. 1987a, ApJS, 63, 947

Hillier, D.J. 1987b, ApJS, 63, 965

Hillier, D.J. 1988, ApJ 327, 822

Ignace, R., Brown, J.C., Milne, J.E., Cassinelli, J.P. 1998, A\&A, 337, 223

Koenigsberger, G., Auer, L.H. 1985, ApJ 297, 255

Kron, G.E., Gordon, K.C. 1950, ApJ 111, 454

Kuhi, L. 1973, in: M.K.V. Bappu \& J. Sahade (eds.), Wolf-Rayet Stars and HighTemperature Stars, Proc. IAU Symp. No. 49 (Dordrecht: Kluwer), p. 59

Marchenko, S.V., Moffat, A.F.J, 1998a, A\&A 341, 211

Marchenko, S.V., Moffat, A.F.J. 1998b, ApJ 499, L195

Marchenko, S.V., Moffat, A.F.J, Eenens, P.R.J., et al. 1997, ApJ 485, 826

Marchenko, S.V., Moffat, A.F.J, Eversberg, T., et al. 1998, MNRAS 294, 642

Marchenko, S.V., Moffat, A.F.J, Koenigsberger, G. 1994, ApJ 422, 810

Moffat, A.F.J., Marchenko, S.V. 1996, A\&A 305, L29

Morel, T., Marchenko, S., Eenens, P.R.J, et al. 1999, ApJ in press

Morel, T., St-Louis, N., Marchenko, S. 1997, ApJ 482, 470

Morel, T., St-Louis, N., Moffat, A.F.J., et al. 1998, ApJ 498, 413

Münch, G. 1950, ApJ 112, 266

Pauldrach, A., Puls, J., Hummer, D.G., Kudritzki, R.P. 1985, A\&A L1

Robert, C., Moffat, A.F.J., Bastien, P., St-Louis, N., Drissen, L. 1990, ApJ 359, 211

Rochowicz, K. 1997, Acta Astr. 47, 381

Rodrigues, C.V., Magalhães, A.M. 1995, in: K.A. van der Hucht \& P.M. Williams (eds.), Wolf-Rayet Stars: Binaries, Colliding Winds, Evolution, Proc. IAU Symp. No. 143 (Dordrecht: Kluwer), p. 260

Schulte-Ladbeck, R.E. 1994, in: A.F.J. Moffat, S.P. Owocki, A.W. Fullerton, N. St-Louis (eds.), Instability and Variability of Hot-Star Winds, (Dordrecht: Kluwer), p. 347

Schulte-Ladbeck, R.E., Eenens, P.R.J., Davis, K. 1995, ApJ 454, 917

Schmutz, W. 1997, A\&A 321, 268

Springmann, U. 1994, A\&A 289, 505

Steiner, J.E., Diaz, M.P. 1998, PASP 110, 276

Stevens, I.R., Howarth, I.D. 1999, MNRAS 302, 549

St-Louis, N., Dalton, M.J., Marchenko, S.V., Moffat, A.F.J., Willis, A.J. 1995, ApJ 452, L57

St-Louis, N., Moffat, A.F.J., Lapointe, L., et al. 1993a, ApJ 410, 342

St-Louis, N., Willis, A.J., Stevens, I.R. 1993b, ApJ 415, 298

Stone, J.M., Xu, J., Mundy, L.G. 1995, Nature 377, 315

Underhill, A.B., Fahey, R.P. 1987, ApJ 313, 358

\section{Discussion}

Schmutz: I have to congratulate you for your careful observational work and its interpretation. I think this is very important for constraining the theoretical models. However, I would also like to emphasize that the type of variations you showed - even the strong ones of WR 6 - are irrelevant for a spectroscopic analysis. The stellar parameters inferred are not affected significantly. The only difference is that the line fits are a bit worse in some cases. 
Marchenko: This well may be the case, but, as I mentioned for EZ CMa (WR 6), the wind is: (a) flattened, and (b) large-scale structured. As the star rotates (this is currently my most plausible explanation for the observed variations) you detect $\sim 30 \%$ variations in the terminal velocity, at least in the formally defined velocity. This leads to two possible conclusions: (a) there is a significant change in the wind dynamics for a given 'slice' of the structured wind - this I doubt; or (b) there might be a significant change of the ionization structure. I suspect you may deduce somewhat different stellar parameters for given periods of times having that much of epoch-dependent behaviour.

Hillier: You need to be careful when describing changes in the absorption component as reflecting changes in $v_{\infty} . v_{\infty}$ refers to the maximum velocity of the mean flow. From the work of Owocki et al. we know that instabilities generate material at higher velocities. This 'high-velocity' material is believed to give rise to absorption beyond $v_{\infty}$ in UV P-Cygni resonance lines. In addition, the absorption component only samples a small volume of material. In the profiles you showed the emission components exhibited much less variation than the absorption components, indicating that the mean flow was not changing very much.

Marchenko: Demonstrating the variability pattern of EZ CMa, I exaggerated, though consciously, the potential impact of the variations on the modeling of the stellar spectrum. My point is that there are some (well) behaving binaries where the wind can be treated as spherically symmetric, without detectable large-scale structuring. Regarding the $v_{\infty}$ variations: in fact, there are accompanying variations in the emission parts of the profiles, which signifies some modulation in $\dot{M}$, inevitably leading to a modulation of the bulk velocity. However, the $\dot{M}$-modulation is far smaller then the formally defined $\sim 30 \%$ variations in $v_{\infty}$ of EZ CMa. These variations might be connected to a significant change in the ionization structure.

Maeder: You have shown these nice instabilities made by a fast wind hiting a slow wind. Do you mean that the fast and slow winds have been emitted at different epochs and what differences in time are required so that the model is working?

Marchenko: In the original work of Stone et al. (1995) they suggest very large variations in the mechanical luminosity of the wind, i.e., $L \propto \dot{M} v_{\infty}$. What would happen if we have variations of the order of 10-20\% occurring on a time-scale of days or a fraction of day? This was never addressed in modeling. However, we do observe such variations. Extrapolating the model of Stone et al. I expect that we might have a potential possibility to create instable layers at the distances far exceeding the volume of the wind subjected to the radiative instabilities, thus reaching, say, $r \simeq 100 R_{*}$. Additionally, this might help to solve the problem of the tangential/radial discrepancy observed in the velocity dispersion of the wind-formed clumps (Lepine 1998, $\mathrm{PhD}$ thesis).

Koenigsberger: I'm confused by the difficulty you mentioned to have in detecting the broadening of the photosperic absorption lines due to electron scattering. Münch (1950) was able to see this in his photographic spectra of V444 Cygni.

Marchenko: In fact, we have used the Münch's model to fit the profile changes caused by the electron scattering in V444 Cygni. However, the model has been developed neglecting the bulk motion of the scattering medium. If you want to include this bulk motion, you immediately realize that very important information can be extracted looking for the changes in the wide wings of the lines, together with the changes occurring in the core. That demands a very high $\mathrm{s} / \mathrm{N} \gtrsim 500$. 